\title{
Focal Bone Marrow Lesions: A Complication of Ultrasound Diathermy
}

\author{
Seung Jae J. Kim, Yusuhn Kang ${ }^{1}$, Dae Ha Kim², Jae Young Lim³ ${ }^{3}$ Joo Hyun Park, Joo Han Oh \\ Departments of Orthopedic Surgery and ${ }^{1}$ Radiology, Seoul National University Bundang Hospital, Seoul National University College of Medicine, Seongnam, \\ ${ }^{2}$ Department of Orthopedics and Sports Medicine, CM General Hospital, Seoul, ${ }^{3}$ Department of Rehabilitation Medicine, Seoul National University Bundang \\ Hospital, Seoul National University College of Medicine, Seongnam, Korea
}

\begin{abstract}
Ultrasound diathermy is widely used for the treatment of musculoskeletal disorders and other soft tissue injuries. Its use as a therapeutic modality is believed to be safe, with very few reported complications. Here, we report two patients who developed focal bone marrow abnormalities after receiving ultrasound diathermy. Both patients' magnetic resonance (MR) evaluations revealed linear subchondral bone lesions of the superolateral humeral head similar to those in osteonecrosis. The patients' symptoms subsequently improved, and available follow-up MR evaluation revealed near complete resolution of bone lesions. These findings suggest that ultrasound diathermy, and its interaction with bone tissue through thermal mechanisms, can cause focal bone marrow abnormalities. Furthermore, the bone marrow abnormalities seem to be transient, resolving upon cessation of ultrasound diathermy, therefore osteonecrosis should be differentiated from this temporal lesion.
\end{abstract}

(Clin Shoulder Elbow 2019;22(1):40-45)

Key Words: Bone marrow lesion; Osteonecrosis; Magnetic resonance imaging; Complications; Diathermy

Diathermy, the use of energy to raise the temperature of deep soft tissue, is a therapeutic modality that is widely used to treat certain musculoskeletal disorders. The most commonly used forms of energy include ultrasound, shortwave, and microwave." Ultrasound, a high-frequency electrical energy in the form of an inaudible sound wave, is absorbed by tissue resulting in its conversion to heat. ${ }^{1,2)}$ Ultrasound is a commonly used form of diathermy energy in orthopedics, rehabilitation, and physiotherapy. Ultrasound diathermy is recognized as an effective and safe therapeutic modality for soft tissue injuries. ${ }^{1,3)}$ Although the therapeutic properties of ultrasound have been demonstrated clinically, the exact mechanisms of ultrasound interactions with biological tissues are not well understood. ${ }^{2)}$

General heat precautions and contraindications have been described, but there is a lack of information on specific complications of ultrasound diathermy. ${ }^{1)}$ To date, only one report of focal bone abnormalities as a result of ultrasound diathermy has been published. ${ }^{4)}$ The cases described in the current study presented with bone marrow abnormalities after receiving ultrasound diathermy. The purpose of this study is to introduce ultrasound diathermy as a source of focal bone marrow abnormalities and to describe the progression of such lesions.

\section{Case Report}

\section{Patient 1}

A 34-year-old female patient was referred to Seoul National University Bundang Hospital complaining of right shoulder pain. She reported onset of remote pain 1 year ago, and recent aggravation 4 months prior to her visit to our hospital. She had history of two steroid injections and physiotherapy sessions using therapeutic ultrasound diathermy at an outside clinic, and no history

Received August 31, 2018. Revised September 29, 2018. Accepted October 16, 2018.

Correspondence to: Joo Han Oh

Department of Orthopedic Surgery, Seoul National University Bundang Hospital, Seoul National University College of Medicine, 82 Gumi-ro 173 beon-gil, Bundang-gu, Seongnam 13620, Korea

Tel: +82-31-787-7197, Fax: +82-31-787-4056, E-mail: ohjh1@snu.ac.kr, ORCID: https://orcid.org/0000-0002-4962-3175

IRB approval: Seoul National University Bundang Hospital (No. B-1808-487-701).

Financial support: None. Conflict of interests: None. 
of trauma. Exact dosage (intensity) or number of sessions was not available. On physical examination, range of motion (ROM) of the right shoulder was limited with forward flexion (FF), external rotation (ER) at side, and internal rotation (IR) at back of $90^{\circ}$, $15^{\circ}$, and the level of the fourth lumbar vertebrae, respectively. Pain was scored on the visual analogue scale (VAS) at 9.

Plain radiographs revealed minor sclerotic change and no other significant findings (Fig. 1). Magnetic resonance imaging (MRI), performed at an outside clinic before and after physiotherapy sessions, was reviewed. Pre-physiotherapy magnetic resonance (MR) arthrogram revealed supraspinatus bursal fraying. Although MR arthrogram is not optimal for bone marrow imaging, no evidence of bone marrow abnormalities was observed (Fig. 2A). Post-physiotherapy MRI revealed newly developed area of linear subchondral bone marrow abnormalities consistent with osteonecrosis in the superolateral humeral head. The MRI depicted hypointense band-like lesion on the T1-weighted image and hyperintense band-like lesion on T2-weighted fatsuppressed image (Fig. 2B, C). Similar bone marrow lesions were also observed at the superior aspect of the acromion (Fig. 2B, C). The location at areas of thinner overlying soft tissue and the superficial nature of the bone marrow lesions were also taken into consideration. MRI abnormalities, confirmed by an experienced musculoskeletal radiologist, suggested osteonecrosis of the humeral head combined with a clinical diagnosis of frozen shoulder. Additional investigation of clinical history for predisposing factors for osteonecrosis, such as trauma, corticosteroid use, alcoholism, and coagulative blood disorders, revealed no significant findings.

Nonsteroidal anti-inflammatory drugs (NSAIDs, zaltoprofen) were prescribed, and a stretching program was implemented to address frozen shoulder. She was followed-up regularly, and there was no evidence of progression of osteonecrosis. In fact, she showed marked improvements in ROM and pain. By 14 months after the initial visit, she had progressed to near full ROM of FF, ER at side, and IR at back of $165^{\circ}, 80^{\circ}$, and the level of the seventh thoracic vertebrae, respectively, and pain VAS of 0. Follow-up MRI examination, performed at 13 months after ini-
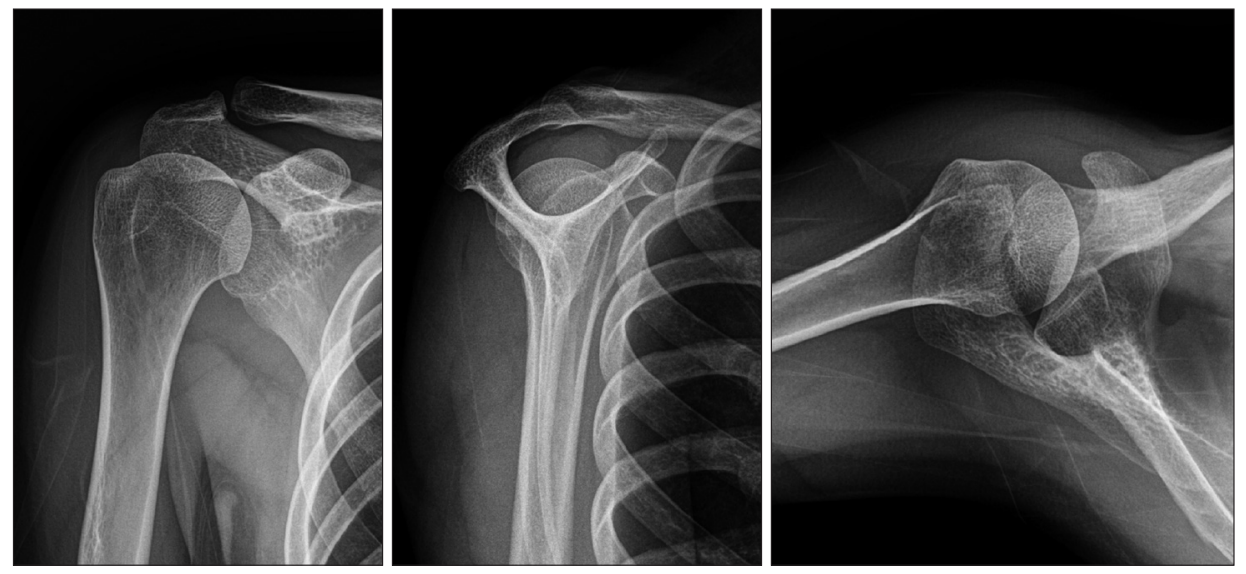

Fig. 1. Simple radiographys of patient 1 reveal minor sclerotic changes of shoulder joint.
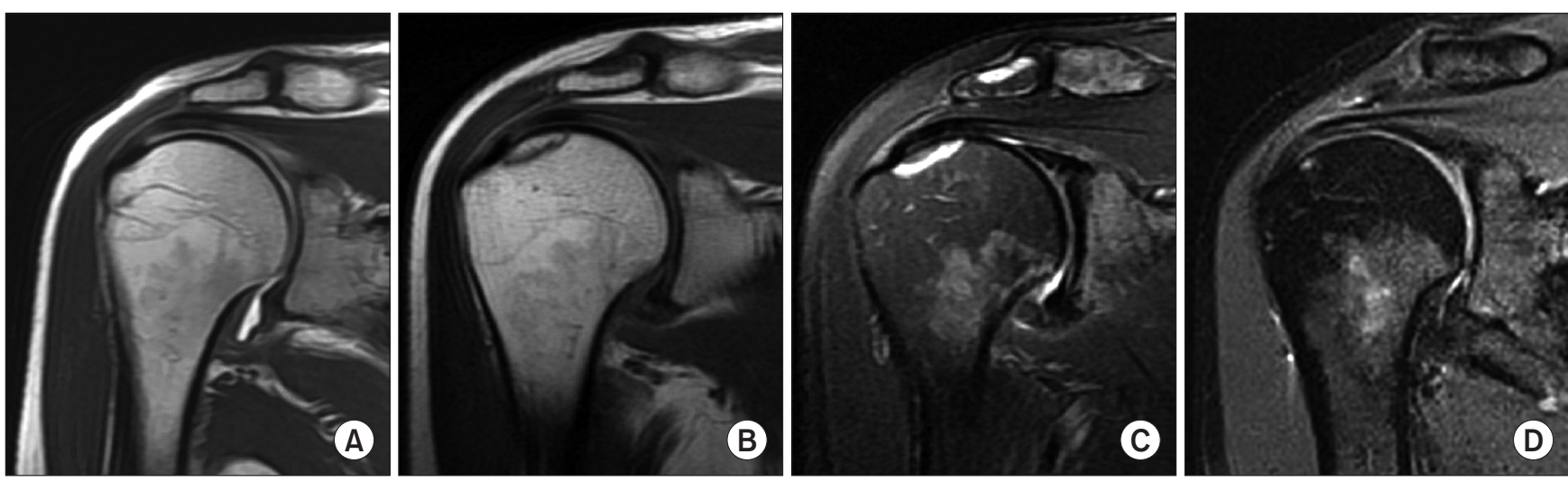

Fig. 2. Serial magnetic resonance (MR) examinations of patient 1 show development and near-complete resolution of focal bone marrow abnormalities after ultrasound diathermy. (A) Pre-physiotherapy MR arthrogram T1-weighted image shows no definite abnormality in the humeral head. Post-physiotherapy MR imaging (MRI) T1-weighted image shows hypointense band-like lesion (B), and T2-weighted fat-suppressed image shows hyperintense band-like lesion at right superolateral humeral head (C). Similar bone lesions are seen at superior surface of the acromion. Notably, all lesions are superficially located. (D) Follow-up proton density fat-suppressed MRI performed 13 months later shows near complete resolution of the lesions. 
tial visit, revealed near complete resolution of previously noted bone marrow lesions at the superolateral humeral head and superior acromion (Fig. 2D). Due to unnatural improvement of MR findings and clinical history of no known predisposing factors, osteonecrosis of the humeral head was determined to be less likely. We concluded that the initial bone lesions observed on MRI were likely focal bone marrow abnormalities resulting from ultrasound diathermy.

\section{Patient 2}

A 54-year-old female patient was referred to our hospital complaining of left shoulder pain, coinciding with increased golf play 5 months prior. The patient had a history of multiple physiotherapy sessions using ultrasound diathermy at an outside clinic and no history of trauma. Exact dosage or number of sessions was not confirmed. On physical examination, ROM of shoulders was unrestricted, and findings on the left shoulder were positive for compression rotation test, Whipple test, O'Brien test, and AbER test, suggesting a superior labrum anterior and posterior (SLAP) lesion.

Plain radiographs revealed left subacromial spur and no significant findings on the humeral head (Fig. 3A). MR arthrogram revealed subchondral bone marrow abnormalities suggestive of osteonecrosis in the superolateral humeral head. Hyperintense subchondral bone marrow lesion on T1-weighted image and hypointense band-like lesion on T2-weighted image were observed (Fig. 4A, B). The location and superficial nature of the lesion were taken into consideration. No bone marrow lesions were observed in the acromion. Other abnormal MR arthrogram findings included type 2 SLAP lesion and partial thickness tear of the subscapularis muscle. Bone scan revealed increased uptake in the left humeral head, anatomically coinciding with bone lesions seen in MRI (Fig. 4C). MR arthrogram abnormalities, confirmed by the radiologist, were suggestive of osteonecrosis of the humeral head, combined with shoulder impingement and type 2 SLAP lesion. Additional investigation of clinical history for predisposing factors for osteonecrosis revealed no significant findings. Subacromial ultrasonography (USG)-guided steroid injection, NSAIDs (zaltoprofen), and stretching program were prescribed. She was followed-up regularly and displayed improvement of pain symptoms at 3 months and 8 months. However, patient was lost to follow-up and immediate follow-up MR examination is unavailable.

Five years after initial visit, she returned to clinic complaining of left shoulder pain. She displayed unrestricted ROM and pain VAS of 7. She reported history of shoulder injury from weight
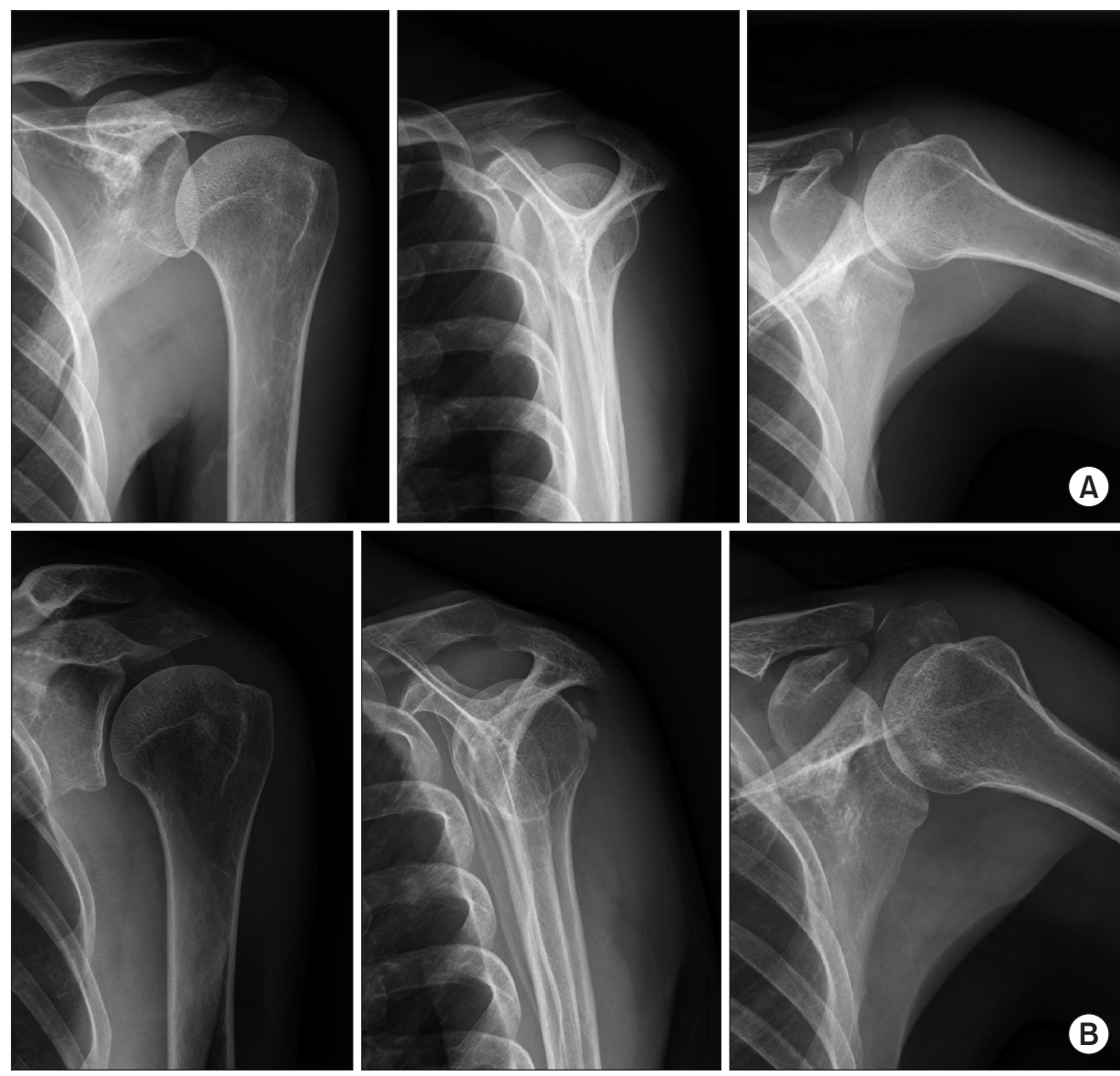

Fig. 3. (A) Simple radiography of patient 2 reveals left subacromial spur. (B) Simple radiography at return to clinic reveals calcification deposit adjacent to the left greater tubercle. 

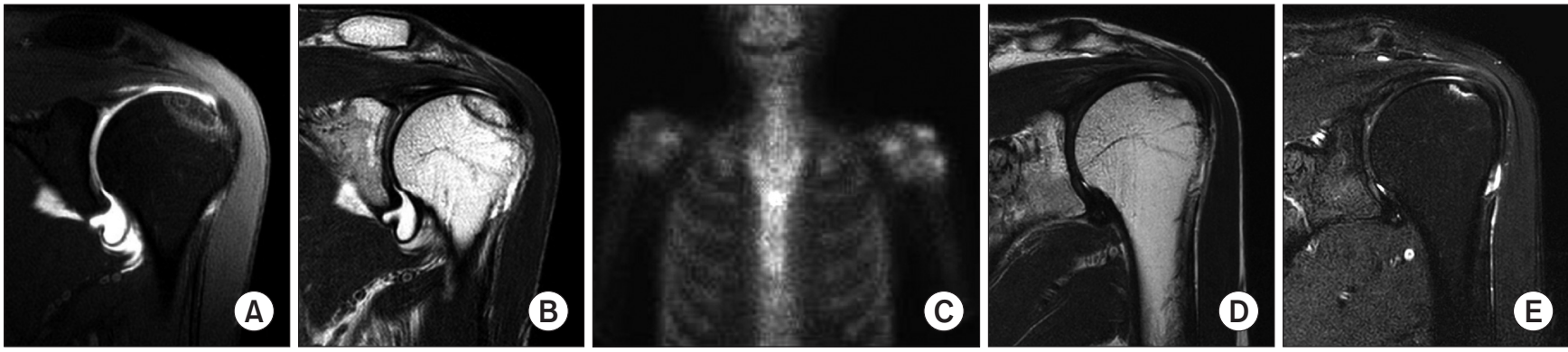

Fig. 4. Magnetic resonance (MR) examinations of patient 2 show development and improvement of focal bone marrow abnormalities after ultrasound diathermy. Post-physiotherapy MR arthrogram (A) T1-weighted image shows hyperintense subchondral bone marrow lesion, and (B) T2-weighted image shows hypointense band-like lesion at left superolateral humeral head. (C) Bone scan reveals increased uptake in the left humeral head. Second MR imaging examination, taken 5 years later, reveals (D) hypointense band-like lesion on T2-weighted image, and (E) hyperintense band-like lesion on T2-weighted fat suppressed image. Again, all lesions are located superolaterally and superficially.

exercise and history of two steroid injections and physiotherapy sessions at an outside clinic. Plain radiographs revealed calcification deposit adjacent to the left greater tubercle and no other significant findings (Fig. 3B). MRI examination revealed near complete resolution of previous osteonecrosis-like bone marrow lesions at the superolateral humeral head. Hypointense bandlike lesion on T2-weighted images and hyperintense band-like lesion on T2-weighted fat suppressed images were detected (Fig. $4 \mathrm{D}, \mathrm{E})$. The superolateral location and superficial nature were again noted. Other abnormal findings included type 2 SLAP lesion and tendinopathy of supraspinatus and subscapularis tendons. Subacromial USG-guided steroid injection and stretching program were prescribed. She was followed-up regularly and showed improvement in pain symptoms. However, followup MRIs are again unavailable because the patient was lost to follow-up.

\section{Discussion}

Focal bone marrow abnormalities after ultrasound diathermy are a complication that has not previously been fully described. Review of past literature revealed only one study on focal bone abnormality as a complication of ultrasound diathermy. Yeh et al." described eight patients who developed marrow lesions similar to those of focal osteonecrosis at various joints after undergoing physiotherapy using ultrasound diathermy. Pre-ultrasound diathermy procedure MR image evaluations were not available in this study.

Our report described two patients with ultrasound diathermyassociated focal bone marrow abnormalities of the superolateral humeral head. Pre-procedure, post-procedure, and follow-up MR evaluation of patient 1 showed development and subsequent near-complete resolution of osteonecrosis-like lesions of the humeral head. Post-procedure MR evaluation of patient 2 revealed development of focal bone marrow abnormalities of the humeral head, and MR evaluation 5 years after initial visit also showed small residual bone marrow lesions. Although it is difficult to completely rule out the possibility of idiopathic osteonecrosis of the humeral head, the timing and transient nature of the findings in relation to diathermy procedures suggest that ultrasound diathermy is the cause of these focal bone marrow lesions.

Ultrasound is used as a therapeutic modality through both its thermal and nonthermal mechanisms. The therapeutic uses of ultrasound mechanisms are dependent on specific techniques and parameters, such as frequency, intensity, and delivery method. ${ }^{1,5)}$ A circular or longitudinal stroking technique is needed for even energy distribution. Frequency ranges between 0.8 to 3.0 $\mathrm{MHz}$, with high frequencies used for superficial areas and low frequencies used for better penetration. There are no specific guidelines for output, and intensity is adjusted just below the pain threshold. Ultrasound delivery can be continuous or pulsed, depending on whether a thermal or nonthermal effect is preferable. In acute conditions, lower intensity pulsed-wave ultrasound is preferred for nonthermal effects to reduce edema. In chronic conditions, higher intensity continuous-save ultrasound is preferred for thermal effects to increase blood flow, temperature, and flexibility.

Ultrasound diathermy treatment is a widely used form of noninvasive therapy in the field of orthopedics and physiotherapy for softening scar tissue, resolving edema, and accelerating wound healing. ${ }^{5,6)}$ A randomized controlled trial demonstrated significant benefits of ultrasound therapy in resolving calcific deposits and improving pain symptoms in patients with calcific tendinitis. ${ }^{3)}$ However, conflicting reports on the benefits of ultrasound diathermy and a dearth in clinical trials regarding its use have resulted in a lack of information, no specific indications for treatment, and empirically determined treatment parameters. ${ }^{1,6)}$

Contraindications include use near structures vulnerable to heat, such as nerves, brain, eyes, and reproductive organs, malignancies, infections, pregnancy, and metallic implants." Previous studies observed no reported side effects of ultrasound 
diathermy. ${ }^{3)}$ Proper stroking technique and accurate frequency for target tissue depth are required to avoid local hotspots, periosteal damage, and acoustic cavitation. ${ }^{1)}$ Draper et al. ${ }^{7)}$ recommended $1 \mathrm{MHz}$ ultrasound for heating tissues 2.5 to $5.0 \mathrm{~cm}$ deep, and $3 \mathrm{MHz}$ ultrasound for heating tissues less than $2.5 \mathrm{~cm}$ deep. However, a recent survey of physical therapists on usage trends of therapeutic ultrasound revealed a lack of knowledge of absolute contraindications and of basic theory regarding physiological effects and its interaction with biological tissues. ${ }^{5}$ This lack of knowledge can lead to ineffective treatment, and more importantly, could be potentially harmful to patients.

Proper knowledge of basic ultrasound mechanisms and biological interactions is essential to understanding adverse effects and potential thermal damage to the bone. Focused ultrasound directed at rabbit femurs resulted in significant thermal damage to bone, characterized by histological evidence of completely empty lacunae and MRI evidence of damage and edema of the bone. ${ }^{8)}$ It was hypothesized that development of osteonecrosislike focal bone marrow changes seen in MRIs of human patients could be related to ultrasound diathermy treatments. ${ }^{4)}$ Similarly, osteonecrosis of humeral head was reported in a patient after receiving extracorporeal shock-wave therapy for rotator cuff tendinopathy."

MRI is an important tool for early diagnosis of osteonecrosis. The diagnostic MRI signs of osteonecrosis of the humeral head include linear bands of low intensity on both T1- and T2-weighted images and double-line sign on T2-weighted images. These lesions are located at the superomedial portion of the humeral head. Our patients displayed osteonecrosis-like lesions, characterized by an area of linear band-like subchondral bone marrow abnormalities of the humeral head. However, the superficial nature and location of the marrow lesions are worth consideration. All lesions were found at areas near thin overlying soft tissue, such as the superolateral portion of the humeral head and at the superior side of the acromion. Using MR examinations, both patients were initially assessed for osteonecrosis of the humeral head. Although MRI achieves excellent sensitivity, due to the inherent nature of the test, there is a high false-positive rate. ${ }^{10)}$

This suggests the importance of clinical information in the diagnosis of osteonecrosis. Common etiologies include posttraumatic sequelae, corticosteroid use, and alcoholism. Osteonecrosis of the humeral head is not only incredibly rare, but is frequently asymptomatic until the head collapses due to the lack of weight bearing burden at the shoulder joint. ${ }^{10)}$ Furthermore, osteonecrosis progression has not been shown to be reversible. Both patient 1 and patient 2 revealed no clinical history of predisposing factors. Also, both patients displayed symptoms of shoulder pathology that correlated with pre-existing shoulder disorders prior to ultrasound diathermy therapy. Both patients were treated for their pre-existing shoulder disorders (frozen shoulder in patient 1 , and shoulder impingement and SLAP le- sion in patient 2) rather than for osteonecrosis. Accordingly, both patients experienced symptom relief, but it is difficult to determine if this outcome is due to improvement of the pre-existing shoulder disorder or improvement of bone marrow lesions on MRI.

In a previous report of osseous injury after ultrasound diathermy, resolution of such lesions was observed on cessation of therapeutic ultrasound treatment. ${ }^{4)}$ Smith et al. ${ }^{8)}$ described the process of bone healing after ultrasound thermal damage. Bone healing occurred from the periosteum and vascular ingrowth from adjacent living cortical bone, suggesting that thermal damage to bone tissue will ultimately heal itself. Conversely, humeral head osteonecrosis after extracorporeal shock-wave treatment did not heal, progressing until it eventually required surgical intervention. It was hypothesized that development of irreversible osteonecrosis was caused by microvascular damage due to the nonthermal mechanism of acoustic cavitation. ${ }^{9)}$ Assuming proper technique and intensities based on recommended range were used, cavitation is not the likely cause of the bone damage seen in our patients.

Patient 1, on follow-up MR evaluation, demonstrated near complete resolution of bone marrow abnormalities at 13 months after ultrasound diathermy procedure. These results are similar to those of previous observations of resolution of lesions within 12 months. ${ }^{4}$ However, patient 2 had small, residual focal bone marrow lesions in MR examinations taken 5 years after initial visit. Citing the corresponding locations of the lesions, we concluded that the residual lesions were improving bone marrow abnormalities observed 5 years prior. Although the bone marrow lesions showed significant improvement, contrary to previous reports, the lesions had not completely resolved even after 5 years. Further follow-up MRI is needed to confirm resolution of bone marrow abnormalities in both patients.

This study has several limitations. Both patients presented with symptoms related to prior shoulder pathology, making it difficult to determine the relationship between patient symptoms and observed marrow lesions. Although pre-physiotherapy, postphysiotherapy, and follow-up MR examinations were available for patient 1 , only post-physiotherapy and 5-year follow-up MR examinations were available for patient 2. Due to lack of additional follow-up MR examinations of both patients, it is difficult to confirm the exact timing of complete resolution of bone marrow lesions.

In conclusion, the two cases described demonstrate the development of focal bone marrow abnormalities after ultrasound diathermy. Serial MR examination findings suggest a relationship between the development and improvement of bone abnormalities and ultrasound diathermy therapy. Although bone marrow abnormalities showed significant improvement, residual lesions were observed even after 5 years. Review of these findings emphasizes the importance of accurate clinical history taking and 
consideration of ultrasound diathermy history as a cause of focal bone marrow abnormalities mimicking osteonecrosis. This temporal lesion must be differentiated from osteonecrosis, due to its unique clinical course and the need for a different treatment plan.

\section{References}

1. Chen WS, Annaswamy TM, Yang W, Wang TG. Physical agent modalities. In: Cifu DX, ed. Braddom's physical medicine and rehabilitation. 5th ed. Philadelphia (PA): Elsevier; 2016. 37881.

2. Dalecki D. Mechanical bioeffects of ultrasound. Annu Rev Biomed Eng. 2004;6:229-48. doi: 10.1016/j.jos.2016.12.028.

3. Ebenbichler GR, Erdogmus $\mathrm{CB}$, Resch $\mathrm{KL}$, et al. Ultrasound therapy for calcific tendinitis of the shoulder. N Engl J Med. 1999;340(20):1533-8. doi: 10.1056/NEJM199905203402002.

4. Yeh LR, Chen CK, Tsai MY, Teng HC, Lin KL. Focal bone abnormality as a complication of ultrasound diathermy: a report of eight cases. Radiology. 2011;260(1):192-8. doi: 10.1148/ radiol.11101301.

5. de Brito Vieira WH, Aguiar KA, da Silva KM, Canela PM, da Silva FS, Abreu BJ. Overview of ultrasound usage trends in orthopedic and sports physiotherapy. Crit Ultrasound J. 2012;4(1):11. doi: 10.1186/2036-7902-4-11.

6. ter Haar G. Therapeutic applications of ultrasound. Prog Biophys Mol Biol. 2007;93(1-3):111-29. doi: 10.1016/ j.pbiomolbio.2006.07.005.

7. Draper DO, Castel JC, Castel D. Rate of temperature increase in human muscle during $1 \mathrm{MHz}$ and $3 \mathrm{MHz}$ continuous ultrasound. J Orthop Sports Phys Ther. 1995;22(4):142-50. doi: 10.2519/jospt.1995.22.4.142.

8. Smith NB, Temkin JM, Shapiro F, Hynynen K. Thermal effects of focused ultrasound energy on bone tissue. Ultrasound Med Biol. 2001;27(10):1427-33. doi: 10.1016/S03015629(01)00454-9.

9. Liu HM, Chao CM, Hsieh JY, Jiang CC. Humeral head osteonecrosis after extracorporeal shock-wave treatment for rotator cuff tendinopathy. A case report. J Bone Joint Surg Am. 2006;88(6):1353-6. doi: 10.2106/JBJS.E.00868.

10. Sugano N. Osteonecrosis of the humeral head. In: Koo KH, Mont MA, Jones LC, eds. Osteonecrosis. Berlin: Springer; 2014. 389-94. 